\title{
Local impedance and ultra-high density 3-dimensional mapping results in improved ablation metrics for cavotricuspid isthmus dependent atrial flutter compared with conventional ablation and contact force-guided ablation with 3-dimensional mapping
}

\author{
Karan Saraf ${ }^{1}$, Nicholas Black ${ }^{2}$, Clifford Garratt ${ }^{1}$, Sahrkaw Muhyaldeen ${ }^{2}$, and Gwilym \\ Morris $^{1}$ \\ ${ }^{1}$ The University of Manchester \\ ${ }^{2}$ Manchester University NHS Foundation Trust
}

January 14, 2021

\begin{abstract}
Introduction Multiple contact-based ablation technologies have been developed to allow real-time judgement of lesion effectiveness; local impedance (LI) guided ablation and the role of ultra-high density (UHD) mapping have not yet been evaluated for cavotricuspid isthmus dependent atrial flutter (CTI-AFL). Methods This non-randomised observational study evaluated patients undergoing CTI-AFL ablation using conventional, contact force (CF) and LI guided strategies. Ablation metrics were collected, and in the LI cohort, the use of UHD mapping for breakthrough was evaluated. Results 30 patients were included, 10 in each group. Mean total ablation time was significantly shorter with LI (3.2 $\pm 1.3 \mathrm{~min})$ vs conventional $(5.6 \pm 2.7 \mathrm{~min})$ and $\mathrm{CF}(5.7 \pm 2.0 \mathrm{~min}, \mathrm{p}=0.0042)$. Time from start of ablation to CTI block was numerically shorter with LI (14.2 $\pm 8.0 \mathrm{~min})$ vs conventional and $\mathrm{CF}(19.7 \pm 14.1$ and $22.5 \pm 19.1 \mathrm{~min}, \mathrm{p}=0.4408)$. There were no differences in the number of lesions required to achieve block, procedural success, complication rates or recurrence. 15/30 patients did not achieve block following first-pass ablation. UHD mapping rapidly identified breakthrough in the 5 LI patients, including epicardial-endocardial breakthrough (EEB) away from the line. Conclusion The use of LI for real-time assessment of lesion formation resulted in significantly less ablation requirement. UHD mapping rapidly identified breakthrough, including EEB, which would likely have been difficult to identify otherwise and possibly require extensive ablation, contributing towards shortening of time to CTI block with LI.
\end{abstract}

\section{Hosted file}

LI paper final 06-01-21.pdf available at https://authorea.com/users/389372/articles/503987local-impedance-and-ultra-high-density-3-dimensional-mapping-results-in-improvedablation-metrics-for-cavotricuspid-isthmus-dependent-atrial-flutter-compared-withconventional-ablation-and-contact-force-guided-ablation-with-3-dimensional-mapping 